TRANSACTIONS OF THE

AMERICAN MATHEMATICAL SOCIETY

Volume 358 , Number 4 , Pages 1657-1675

S 0002-9947(05)03760-8

Article electronically published on August 1, 2005

\title{
HEIGHT UNIFORMITY FOR INTEGRAL POINTS ON ELLIPTIC CURVES
}

\author{
SU-ION IH
}

\begin{abstract}
We recall the result of D. Abramovich and its generalization by P. Pacelli on the uniformity for stably integral points on elliptic curves. It says that the Lang-Vojta conjecture on the distribution of integral points on a variety of logarithmic general type implies the uniformity for the numbers of stably integral points on elliptic curves. In this paper we will investigate its analogue for their heights under the assumption of the Vojta conjecture. Basically, we will show that the Vojta conjecture gives a naturally expected simple uniformity for their heights.
\end{abstract}

\section{INTRODUCTION}

There have been several astonishing uniformity results on the number of (rational or integral) points on curves recently. The first one is L. Caporaso, J. Harris, and B. Mazur's uniformity for the number of rational points on curves of genus $\geq 2$ ([ 6$]$ ), which is based on the Lang conjecture on the distribution of rational points on varieties of general type and which was generalized by D. Abramovich ([1]) and further by P. Pacelli (21]) at a later date. The Lang conjecture claims that the set of rational points on a variety of general type cannot be Zariski dense; by a (projective) variety of general type we mean that (one, hence every, desingularization of) the variety has regular pluri-canonical forms enough to give rise to a birational map into a projective space. The next results directly related to this paper are Abramovich's ([2]) and its generalization by Pacelli ([22]), which concern the uniformity for stably integral points on elliptic curves and which are based on the Lang-Vojta conjecture.

Whenever we have finiteness results of rational or integral points on varieties in arithmetic geometry, the effectivity, i.e., the problem of determining all such points or that of giving an explicit upper bound for their heights always becomes at issue. Along this line, the author (15]) previously gave a height version of the above result of Pacelli in 21. before. In this paper he will give a height version of Pacelli's result in 22. However, contrary to Abramovich's and Pacelli's study, it is worth noticing that we can take all integral points (not necessarily stably integral) into consideration in our study of their heights.

In the early 1980's P. Vojta discovered an uncanny similarity between Diophantine approximation theory and Nevanlinna theory (value distribution theory for

Received by the editors March 6, 2004 and, in revised form, June 9, 2004.

2000 Mathematics Subject Classification. Primary 11G35, 11G50, 14 G05.

Key words and phrases. Ample divisor, big divisor, canonical divisor, height, height zeta function, symmetric product, variety of general type, Vojta conjecture.

(C)2005 American Mathematical Society Reverts to public domain 28 years from publication 
complex analytic functions) - the fact is that it should be noted that C. Osgood ([19]) has also, previously, noticed a Nevanlinna-Roth connection. This great insight of his led him to his main conjecture and his independent proof of the Mordell conjecture (31]). We will precisely state his conjecture later.

Let us state our main result in this paper.

Let $\pi: X \rightarrow Y$ be a family of elliptic curves, i.e., both $X$ and $Y$ are nonsingular projective varieties (of arbitrary dimension), the relative dimension of the surjective morphism $\pi$ is equal to 1 , and all the 1-dimensional nonsingular fibers are elliptic curves - the generic fiber is an elliptic curve. Assume, in addition, that $X, Y$, and $\pi$ are all defined over a number field $k$ and let $S$ be a finite set of primes of $k$ containing all the infinite ones.

Let $d \geq 1$ be an integer and let $D$ be an ample divisor (assumed to be effective) of $X$ with normal crossings. (Hence, in particular, it is not fibral, i.e., $\pi(\operatorname{Supp} D)=$ $Y$.) Denote by

$$
\mathcal{R} \subset X(k, d)-\operatorname{Supp} D
$$

a subset of $(S, D)$-integral points of $X(\bar{k})$ which belong to $X(k, d)(:=$ the subset of points (of $X(\bar{k})$ ) of degree $\leq d$ over $k$ ), hence, in fact $\mathcal{R} \subset X(k, d)-\operatorname{Supp} D$ by definition. Finally choose an arbitrary height $h$ on $X$ and a height $h_{Y}$ on $Y$ associated to an ample divisor satisfying $h_{Y} \geq 1$. (In this article a height is always a Weil height, whose definition is given in the next section.)

Theorem 1.0.1. In addition, assume the Vojta conjecture for varieties of dimension $\leq d \cdot \operatorname{dim} X$. Then there is a constant $c>0$ such that

$$
h(P) \leq c \cdot h_{Y}(\pi(P))
$$

for all $P \in \mathcal{R}$, i.e., $P$ is an $\left(S_{k(\pi(P))}, D_{\pi(P)}\right)$-integral point of $X_{\pi(P)}(\overline{k(\pi(P))})$ belonging to $X_{\pi(P)}(k(\pi(P)))$, where $\pi(P)$ has degree $\leq d$ over $k$ and the fiber of $\pi$ over $\pi(P)$ is an elliptic curve. Note that the constant $c$ is independent of $P$. (In addition, here $S_{k(\pi(P))}$ is the finite set of primes of the number field $k(\pi(P))$ lying over (the primes of) $S$ and $D_{\pi(P)}$ is the restriction of $D$ to the fiber $X_{\pi(P)}$.)

Remark 1 . We can reformulate the result in the following more suggestive way:

$$
h \leq O\left(h_{Y}(t)\right)
$$

on the subset of $\left(S_{k(t)}, D_{t}\right)$-integral points of $X_{t}(\overline{k(t)})$ belonging to $X_{t}(k(t))$, where $t \in Y(\bar{k})$ has degree $\leq d$ over $k$ and the fiber $X_{t}$ of $\pi$ over $t$ is an elliptic curve. Note that the implied constant is independent of $t$.

2. If we drop the hypothesis $h_{Y} \geq 1$, then the above result is reformulated as

$$
h(P) \leq c_{1} \cdot h_{Y}(\pi(P))+c_{2},
$$

where $c_{1}$ and $c_{2}$ are constants $>0$ independent of $P$. This will be clear from the proof.

First, note that we do not have to assume the existence of a rational point on an elliptic curve in this paper. Thus it may be better to refer to a nonsingular projective curve of genus 1 . However, we will still stick to the use of the term "elliptic curve." Second, also note, contrary to Abramovich's ([2]) and Pacelli's (22]) results, that we do not assume that the integral points under consideration in Theorem 1.0.1 are stably integral. Third, precisely speaking, we can suppress $k(\pi(P))$ in the notation of $S_{k(\pi(P))}$; we then naturally consider the finite set of primes of an extension field 
which lie over $S$. And yet, we will sometimes use it for consistency with other people's notation.

Later we will add some more cases for which the height uniformity can be proved in a similar fashion. By the way, we will use the phrase height uniformity for the type of results on the comparison of heights appearing in the theorem above. Indeed, contrary to the case of the number of points, when it comes to height, we clearly cannot expect "genuine" uniformity in general. So there should be no objection to the choice of our term.

As an application of the result above we will introduce in the last section the so-called height zeta function associated to a family studied in Theorem 1.0.1 This is an exact analogue of the height zeta function introduced in [15].

\section{Preliminaries}

2.1. Notation, definitions, and conventions. Unless otherwise stated, by heights we will always mean logarithmic Weil heights. For the general theory of heights we refer to Hindry-Silverman [13, Lang [16, Silverman [28], and Vojta [29]. Here we will give a brief survey of the definition and some basic properties we need later.

Definition. For a point $P:=\left[x_{0}, x_{1}, \ldots, x_{n}\right] \in \mathbb{P}^{n}(\overline{\mathbb{Q}})$, we define the (Weil) height of $P$ to be

$$
h_{\mathbb{P}^{n}}(P)=\frac{1}{d} \cdot \sum_{v} \log \left(\max _{0 \leq i \leq n}\left\{\left|x_{i}\right|_{v}^{d_{v}}\right\}\right),
$$

where $x_{0}, x_{1}, \ldots, x_{n} \in k$ ( $k$ a number field of degree $d$ over $\mathbb{Q}$ ), $v$ runs over (a complete set of) all the primes, finite or infinite, of $k$ so that $|\cdot|_{v}^{d_{v}}$ are normalized (hence satisfying the product formula), and $d_{v}$ is the local degree of $k_{v}$ over $\mathbb{Q} v$.

Remark 1. It is independent of the choice of both the homogeneous coordinates of $P$ and the number field $k$ by the product formula and the contribution of the factor $\frac{1}{d}$, respectively.

2. There is a way called the Weil height machine satisfying various standard properties such as additivity and the functoriality of heights which is to associate a (Weil) height $h_{X, D}: X(\bar{k}) \rightarrow \mathbb{R}$ to a locally principal divisor $D$ on a complete variety $X$ defined over a number field $k$. We do not go into its detail here except to mention the following.

If $D$ is a very ample divisor on $X$, then we define $h_{X, D}:=h_{\mathbb{P}^{n}} \circ \varphi$, where $\varphi: X \hookrightarrow \mathbb{P}^{n}$ is an embedding associated to $D$. More generally, given a locally principal divisor $D$ on $X$, write $D$ as the difference $D_{1}-D_{2}$ of two very ample divisors $D_{i}(i=1,2)$ on $X$. Then we define $h_{X, D}:=h_{X, D_{1}}-h_{X, D_{2}}$, where $h_{X, D_{i}}$ $(i=1,2)$ is as before. Properties of the Weil height machine will be freely used in what follows.

If $X$ is clearly understood from the context, we usually omit $X$ from the notation of $h_{X, D}$. We also sometimes use the linear equivalence class of $D$ or the line bundle $\mathcal{O}_{X}(D)$ instead of $D$ in the notation of $h_{X, D}$. See any reference mentioned above for details.

3. A height $h_{X, D}$ appearing above is uniquely determined only up to a bounded function. Thus, for example, the equality of two heights will always actually mean the equality up to a bounded function. Note that the ambiguity of a bounded function in determining a height does not cause any problem for our usual purposes. 
Later we will often use, in particular, the following property of heights.

Fact [DHA: Dominance of a height associated to an ample divisor] ([16, Chap. 4, Prop. 5.4). Let $h_{c}$ be a height associated to an ample divisor class $c$ with $h_{c} \geq 1$ and $h$ an arbitrary height. Then we have

$$
h=O\left(h_{c}\right) .
$$

Let $d \geq 1$ be an integer. Then, for an arbitrary variety $V$ defined over a number field $k$, let $V(k, d)$ (resp. $V(k,=d))$ be the set of algebraic points on $V$ that have degree $\leq d($ resp. $=d)$ over $k$.

Now let $\pi: X \rightarrow Y$ be a surjective morphism between projective varieties. Assume that $X, Y$, and the morphism $\pi$ are all defined over a number field $k$. Then we define

$$
\begin{aligned}
& Y^{\circ}:=\left\{t \in Y(\bar{k}) \text { : The fiber } X_{t} \text { over } t\right. \text { is nonsingular and has the } \\
& \text { same dimension as that of the generic fiber\}, and } \\
& X^{\circ}:=\bigcup_{t \in Y^{\circ}} X_{t}=\text { The union of nonsingular fibers having the } \\
& \text { same dimension as that of the generic fiber. }
\end{aligned}
$$

Let $\pi: X \rightarrow Y$ be a surjective morphism between projective varieties. For an integer $d \geq 1$, let

$$
X^{(d)}=\operatorname{Sym}_{d} X\left(=X^{d} / S_{d}\right)=\text { the } d^{\text {th }} \text { symmetric product of } X,
$$

where $S_{d}$ is the symmetric group of order $d$ !. Then we have the natural finite surjective morphism

$$
\sigma: X^{d} \longrightarrow X^{(d)}, \quad\left(P_{1}, \ldots, P_{d}\right) \mapsto \sum_{i=1}^{d}\left(P_{i}\right) .
$$

Also, we have the $i^{\text {th }}$ projection $(1 \leq i \leq d) X^{d} \rightarrow X$, and another important induced fibration

$$
\pi^{(d)}: X^{(d)} \longrightarrow Y^{(d)}, \quad \sum_{i=1}^{d}\left(P_{i}\right) \mapsto \sum_{i=1}^{d}\left(\pi\left(P_{i}\right)\right) .
$$

Example 2.1.1. Under the same notation just as above we assume, furthermore, that $X$ is nonsingular. Fix an integer $d \geq 1$ and recall the above natural induced finite surjective morphism $\sigma: X^{d} \rightarrow X^{(d)}$.

Let $W$ be a closed subvariety of $X^{(d)}$. Then we look at the composition $f: \widetilde{W} \rightarrow$ $X^{(d)}$ of $\nu$ and the inclusion $W \hookrightarrow X^{(d)}$, where $\nu: \widetilde{W} \rightarrow W$ is a desingularization. Note that $X^{(d)}$ is normal and projective. Let $H$ be a hyperplane section of $X$ (or an arbitrary ample divisor of $X$ ) and let $h$ be the height of $X$ which is associated to $H$ (not necessarily with the assumption that $h$ be nonnegative). Write

$$
H^{(d)}=\sigma_{*} \sum_{i=1}^{d} \sigma_{i}^{*} H \in \operatorname{Div} X^{(d)},
$$

where $\sigma_{i}: X^{d} \rightarrow X(1 \leq i \leq d)$ is the $i^{\text {th }}$ projection. N.B. There is no danger of confusion with the $d^{\text {th }}$ symmetric product of $H$.

Recall that $\sigma$ is a finite surjective morphism and note that $k\left(X^{d}\right)$ is a finite extension of $k\left(X^{(d)}\right)$. Let $f \in k\left(X^{d}\right)$ be a local equation for the divisor $\sum_{i=1}^{d} \sigma_{i}^{*} H$ 
on $X^{d}$ and let Norm $(f)$ be the norm of $f$, i.e., the determinant of the $k\left(X^{(d)}\right)$ linear endomorphism of $k\left(X^{d}\right)$ given by the multiplication by $f$. Then we know (Proposition $1.4(\mathrm{~b}), 9$ ] $)$ that $\sigma_{*}(\operatorname{div}(f))=\operatorname{div}(\operatorname{Norm}(f))$. Hence $\operatorname{Norm}(f)$ is a local equation for the divisor $H^{(d)}$ on $X^{(d)}$.

Thus $H^{(d)}$ is a locally principal (Weil) divisor, hence to it we can associate a height (by the Weil height machine). We also note that

$$
\sigma^{*} H^{(d)}=c \cdot \sum_{i=1}^{d} \sigma_{i}^{*} H
$$

for some constant $c \geq 1$. Furthermore, it also follows that $H^{(d)}$ is an ample divisor, since $\sigma$ is a finite surjective morphism.

We then have

$$
\begin{aligned}
h_{X^{(d)}, H^{(d)}} \circ \sigma & =h_{X^{d}, \sigma^{*} H^{(d)}}=c \cdot h_{X^{d}, \sum_{i=1}^{d} \sigma_{i}^{*} H} \\
& =c \cdot \sum_{i=1}^{d} h_{X^{d}, \sigma_{i}^{*} H}=c \cdot \sum_{i=1}^{d} h_{X, H} \circ \sigma_{i} \\
& =c \cdot \sum_{i=1}^{d} h \circ \sigma_{i} .
\end{aligned}
$$

Let $\mathcal{P}=\sum_{i=1}^{d}\left(P_{i}\right)=\sigma\left(\left(P_{1}, \ldots, P_{d}\right)\right)$, where $P_{i} \in X$ for $1 \leq i \leq d$. We then note that

$$
\begin{aligned}
h_{X^{(d)}, H^{(d)}}(\mathcal{P}) & =c \cdot \sum_{i=1}^{d} h\left(P_{i}\right) \\
( & \geq 1, \quad \text { if } h \geq 1), \quad \text { and } \\
h_{\widetilde{W}}(\mathfrak{p}) & :=h_{\widetilde{W}, f^{*}\left(H^{(d)}\right)}(\mathfrak{p})=h_{X^{(d)}, H^{(d)}}(f(\mathfrak{p})) \\
& =h_{X^{(d)}, H^{(d)}}(\nu(\mathfrak{p})) \\
( & \left.=c \cdot \sum_{i=1}^{d} h\left(P_{i}\right), \quad \text { if } \nu(\mathfrak{p})=\sum_{i=1}^{d}\left(P_{i}\right)\right)
\end{aligned}
$$

where $\mathfrak{p} \in \widetilde{W}$.

Therefore, if we assume, in addition, that $h \geq 0$, then we have

$$
h(P) \leq O\left(h_{\widetilde{W}}(\mathfrak{p})\right),
$$

where $P=P_{1}$ and $\nu(\mathfrak{p})=\sum_{i=1}^{d}\left(P_{i}\right)$.

On the other hand, if we assume, in addition, that $h \geq 1$ (by noticing $H$ is an (indeed, very) ample divisor) and that $P_{i}$ 's $(1 \leq i \leq d)$ are Galois conjugates of $P=P_{1}$, then we have

$$
h_{X^{(d)}}\left(\sum_{i=1}^{d}\left(P_{i}\right)\right) \leq O(h(P)),
$$

where $h_{X^{(d)}}:=h_{X^{(d)}, H^{(d)}}$ (which is then $\geq 1$ ). (In particular, to $Y$ of a family $X \rightarrow Y$ we will apply what we stated above.) 
Example 2.1.2. We assume $X, Y$, and $\pi$ are all defined over a number field $k$ and let $t^{(1)}=t, t^{(2)}, \ldots, t^{(d)}$ be the $d$ distinct $\operatorname{Gal}(\bar{k} / k)$-conjugates of $t \in Y(k,=d)$. Let $P \in X_{t}(k(t))$. (Here $X_{t}$ denotes the fiber over $t$ under the morphism $\pi$.) Then for all $\sigma \in \mathrm{Gal}(\bar{k} / k), \pi\left(P^{\sigma}\right)=(\pi(P))^{\sigma}=t^{\sigma}$. Thus, by the hypothesis that $P \in X_{t}(k(t))$, we know $P$ has exactly $d$ distinct Gal $(\bar{k} / k)$-conjugates. So $P \in X(k,=d)$. (Indeed, conversely, if $P \in X(k, d) \cap X_{t}$, then $P \in X_{t}(k(t))$ (and hence, $P$ also has exact degree $d$ over $k$ ), where $t$ is as above. This is immediate from a simple computation of degrees of field extensions. Note $\pi$ is defined over $k$.)

2.2. The Vojta Main Conjecture. This will play a crucial role in our subsequent work.

Conjecture 2.2.1 (The Vojta (Main) Conjecture). Let $X$ be a nonsingular complete variety with canonical divisor $K$ and let $D$ be a divisor with normal crossings of $X$, all defined over a number field $k$. Let $S$ be a finite set of primes of $k$ containing all the infinite ones. Then, if $\epsilon>0$ and $A$ is a big divisor of $X$, then there exists a proper Zariski closed subset $Z=Z(X, D, k, S, A, \epsilon)$ of $X$ such that for all $P \in X(k)-Z$,

$$
\sum_{v \in S} \lambda_{D, v}(P)+h_{K}(P) \leq \epsilon h_{A}(P)+O(1) .
$$

(Refer to [13], 16], or [29] for the definition of local heights or Weil functions $\lambda_{D, v}$. Also, refer to [29, 3.4.3, for the details of the conjecture.)

2.3. Preliminary work. In this section we will introduce some preliminary results which will be used later and which may also be interesting in their own right.

Proposition 2.3.1. Let $\pi: X \rightarrow Y$ be a surjective morphism between projective varieties. Then there exist desingularizations $\widetilde{X} \rightarrow X$ and $\widetilde{Y} \rightarrow Y$, and an induced morphism $\tilde{\pi}: \widetilde{X} \rightarrow \widetilde{Y}$ such that $\widetilde{X} \stackrel{\widetilde{\pi}}{\rightarrow} \widetilde{Y} \rightarrow Y$ is equal to $\widetilde{X} \rightarrow X \stackrel{\pi}{\rightarrow} Y$. Furthermore, when $X, Y$, and $\pi$ are all assumed to be defined over a number field $k$, we may assume, by enlarging $k$ if necessary, that all the varieties and the morphisms here are also defined over $k$.

Proof. Let $\widetilde{Y}=$ a desingularization of $Y$ which is the blow-up of $Y$ with respect to a coherent sheaf $\mathcal{I}$ of ideals on $Y, X_{\pi^{-1} \mathcal{I} \cdot \mathcal{O}_{X}}=$ the blow-up of $X$ with respect to the inverse image ideal sheaf $\pi^{-1} \mathcal{I} \cdot \mathcal{O}_{X}$ on $X$, and $\widetilde{X}=$ a desingularization of $X_{\pi^{-1} \mathcal{I} \cdot \mathcal{O}_{X}}$. Then use [11, II 7.15, and we immediately have the desired equality (with induced morphisms). The last statement is trivial. See also Main Theorem I and its Corollary, 14.

Here we want to see an example that should be recalled later - indeed, the inequality of what we are going to prove later is in the opposite direction to that of the following example that is immediate from basic properties of heights.

Example 2.3.2. Let $\pi: X \rightarrow Y$ be a morphism between projective varieties, where $X$ is nonsingular and $Y$ is normal. Also let $h_{Y}$ be an arbitrary height on $Y$ and let $h \geq 1$ be a height on $X$ which is associated to an ample divisor. Let $D$ be a divisor of $Y$ such that $h_{Y}=h_{Y, D}$. Then for all $P \in X$, we have

$$
h_{Y}(\pi(P))=h_{X, \pi^{*} D}(P)+O(1) \leq O(h(P)) \text { by DHA. }
$$

I.e., $O\left(h_{Y} \circ \pi\right) \leq h$ on $X$. Notice this is true of all points of $X$. 
Theorem 2.3.3. Let $X, Y$, and $X_{0}$ be projective varieties, where $X$ is nonsingular and $Y$ is normal. Let $f: X_{0} \rightarrow X$ be a morphism. Suppose that $\pi: X \rightarrow Y$ is a morphism such that $\pi \circ f$ is a generically finite (but not necessarily dominant) morphism. Also, let $h$ and $h_{Y}$ be heights on $X$ and $Y$, respectively, with $h_{Y} \geq 1$ associated to an ample divisor of $Y$. Then,

$$
h \circ f \leq O\left(h_{Y} \circ \pi \circ f\right)
$$

outside a proper Zariski closed subset of $X_{0}$.

Proof. Consider $\varphi: \widetilde{X_{0}} \stackrel{\mu}{\rightarrow} X_{0} \stackrel{f}{\rightarrow} X \stackrel{\pi}{\rightarrow} Y$, where $\mu$ is a desingularization of $X_{0}$. Choose an ample divisor $D$ of $Y$ to which the given height $h_{Y}$ is associated. It will pull back to a big divisor of $\widetilde{X_{0}}$ under $\varphi$. Then, by [29], 1.2.9, (h),

$$
h_{\widetilde{X_{0}}} \leq O\left(h_{\widetilde{X_{0}, \varphi^{*} D}}\right)
$$

outside a proper Zariski closed subset $Z$ of $\widetilde{X_{0}}$, where $h_{\widetilde{X_{0}}} \geq 1$ is a height on $\widetilde{X_{0}}$ associated to an ample divisor. Now apply Example 2.3.2 to see

$$
h \circ f \circ \mu \leq O\left(h_{\widetilde{X_{0}}}\right) \text {. }
$$

Combine these two inequalities and notice that $\mu$ is a birational morphism to get the desired result outside the proper Zariski closed subset $\mu(Z)$ of $X_{0}$.

This is slightly more general than we will use.

2.4. Some observations. Let $P \in X(\bar{k})$ with $\pi(P)=: t \in Y^{\circ}(k,=d)$ and write $i_{t}: X_{t} \hookrightarrow X$ and $D_{t}=i_{t}^{*} D$. Then, $P$ is an $(S, D)$-integral point of $X(\bar{k})$ and belongs to $X(k, d)$ if and only if $P$ is an $\left(S_{k(t)}, D_{t}\right)$-integral point of $X_{t}(\overline{k(t)})$ and belongs to $X_{t}(k(t))$. For $\lambda_{X_{t}, D_{t}, v}=\lambda_{X, D, v}(v$ an arbitrary valuation on $\bar{k})$ on $X_{t}(\bar{k})$, and to see the converse, i.e., that (according to the notation in the statement of Theorem 1.0.1)

$$
P \in X_{t} \cap \mathcal{R} \Longrightarrow P \in X_{t}(k(t))
$$

we also look at

$$
k \subset k^{\prime}=k(\pi(P)) \subset k(P)
$$

with $k^{\prime}:=k(t)$. The last inclusion comes from the hypothesis that $\pi$ be defined over $k$. But, $[k(P): k] \leq d$ and $\left[k^{\prime}: k\right]=d$, so $k^{\prime}=k(P)$ (hence, in particular, $P$ also has degree (over $k$ ) exactly equal to $d$ ). So $k^{\prime}(P)=k^{\prime}$, i.e., $P \in X_{t}(k(t))$ as desired.

2.5. Main proposition. This will play a significant role later.

Proposition 2.5.1. Let $\pi: X \rightarrow Y$ be a surjective morphism between nonsingular projective varieties and let $D$ be a big divisor of $X$ (assumed to be effective). Assume that $X, Y, D$, and $\pi$ are all defined over a number field $k$ and that the generic fiber has canonical divisor which is effective (up to linear equivalence) and which is defined over $k$. Also, let $S$ be a finite set of primes of $k$ containing all the infinite ones.

Now we choose an arbitrary height $h$ on $X$ and a height $h_{Y}$ on $Y$ associated to an ample divisor satisfying $h_{Y} \geq 1$. Then, assuming the Vojta conjecture for varieties of dimension $\leq \operatorname{dim} X$, we have

$$
h(P) \leq O\left(h_{Y}(\pi(P))\right)
$$


for all $(S, D)$-integral points $P \in X(k)-Z$, where $Z$ is a proper Zariski closed subset of $X$ and the implied constant is independent of $P$.

Proof. First, choose an ample divisor $A$ of $X$ (not just a big divisor - for our later use) and then note that the divisor $D+K-\epsilon A$ restricts to a big divisor (up to $\mathbb{Q}$-linear equivalence) on the generic fiber, where $\epsilon>0$ is a small rational number. Use the Kodaira criterion for bigness (Proposition 1.2.7, 29]) to write, up to $\mathbb{Q}$-linear equivalence on the generic fiber, $D+K-\epsilon A$ as the sum of an ample $\mathbb{Q}$-divisor and an effective $\mathbb{Q}$-divisor. Thus $D+K-\epsilon A$ is an effective $\mathbb{Q}$-divisor up to $\mathbb{Q}$-linear equivalence on the generic fiber, since so is the sum. Hence take the Zariski closure in $X$ and there exist a fibral divisor $F$ and an effective divisor $E$ such that

$$
D+K-\epsilon A+F \sim E
$$

in $\operatorname{Div}(X) \otimes \mathbb{Q}$. ("Fibral" means "support not surjecting onto $Y$ under $\pi . ")$

Second, we have the Vojta conjecture for $X$

$$
\sum_{v \in S} \lambda_{D, v}(P)+h_{K}(P) \leq \frac{1}{2} \epsilon h_{A}(P)+O(1)
$$

in $X(k)-Z_{0}$, where $Z_{0}$ is a proper Zariski closed subset of $X$. Here we choose $h_{A}$ to be $\geq 1$ for our later use.

Third, since $F$ is fibral, we can write $F \leq \pi^{*} B$ for some effective divisor $B$ of $Y$. Then we see

$$
\begin{aligned}
h_{F} & \leq h_{X, \pi^{*} B}+O(1) \quad \text { outside Supp } \pi^{*} B \\
& =h_{Y, B} \circ \pi+O(1) \\
& \leq O\left(h_{Y} \circ \pi\right)+O(1) \text { by DHA. }
\end{aligned}
$$

Thus we have

$$
\begin{aligned}
-O(1) & \leq h_{D+K-\epsilon A+F} \text { outside Supp } E \\
& =\left(h_{D}+h_{K}-\frac{1}{2} \epsilon h_{A}\right)-\frac{1}{2} \epsilon h_{A}+h_{F}+O(1) \\
& \leq O(1)-\frac{1}{2} \epsilon h_{A}+O\left(h_{Y}(t)\right)+O(1) \quad \text { with the aid of (4) }
\end{aligned}
$$

in the subset of $\left(S, D_{t}\right)$-integral points of $X_{t}(t \in Y(k))$ that belong to $X_{t}(k)-Z_{1}$, where $Z_{1}=Z_{0} \cup \operatorname{Supp} \pi^{*} B$. By adjusting the implied constants appearing and putting $Z=Z_{1} \cup$ Supp $E$, we then have $h_{A} \leq O\left(h_{Y}(t)\right)$ in the set of those integral points in $X_{t}(k)-Z$ for all $t \in Y(k)$. Therefore we immediately get the desired result by DHA, since $A$ is an ample divisor (class) and $h_{A} \geq 1$ by our hypothesis.

\section{Proof of Theorem 1.0 .1}

\subsection{Lemma for Theorem 1.0.1,}

Lemma 3.1.1. Fix an integer $d \geq 1$. Let $X \rightarrow Y$ be a surjective morphism of relative dimension 1 between projective varieties (of arbitrary dimension) such that all the 1-dimensional nonsingular fibers are elliptic curves. Let $f: X^{(d)} \rightarrow Y^{(d)}$ be the natural induced morphism. Let $t_{1}, \ldots, t_{d}$ be d distinct points of $Y^{\circ}$ and let $\mathcal{T}=$ $\left(t_{1}\right)+\cdots+\left(t_{d}\right) \in Y^{(d)}$. Then (a desingularization of) every positive-dimensional closed subvariety of the fiber over $\mathcal{T}$ under the morphism $f$ has canonical divisor which is (linearly equivalent to) an effective divisor. 
Proof. Observe that the fiber over $\mathcal{T}$ under $f$ is

$$
\left\{\left(P_{1}\right)+\cdots+\left(P_{d}\right) \in X^{(d)}: P_{i} \in X_{t_{i}} \text { for } 1 \leq i \leq d\right\} .
$$

So we may as well be tempted to write formally $X_{t_{1}}+\cdots+X_{t_{d}}$ or $\sum_{i=1}^{d} X_{t_{i}}$ for it, too. We have $\prod_{i=1}^{d} X_{t_{i}} \cong \sum_{i=1}^{d} X_{t_{i}}$ and let $\widetilde{V}$ be a desingularization of a closed subvariety $V$ of dimension $n(\leq d)$ of $\prod_{i=1}^{d} X_{t_{i}}$. We have, by noticing that $X_{t_{i}}$ 's are curves, a surjective morphism

$$
\widetilde{V} \rightarrow V \rightarrow \prod_{i=1}^{n} X_{t_{i}}
$$

after reindexing $X_{t_{i}}$ 's if necessary. Since $\prod_{i=1}^{n} X_{t_{i}}$ has trivial canonical divisor, the canonical divisor of $\widetilde{V}$ comes from just the effective ramification divisor of the morphism.

Fact. Let $X \rightarrow Y$ be a morphism between projective varieties. Assume $X, Y$, and the morphism are all defined over a number field $k$. Also, let $\sigma \in$ Gal $(\bar{k} / k)$ and $t \in Y^{\circ}$ and then also $t^{\sigma} \in Y^{\circ}$. (We can actually say something more: Their (geometric) fibers (called conjugate fibers) are isomorphic, hence, in particular, also $t^{\sigma} \in Y^{\circ}$. However, it is important to notice that this isomorphism is an isomorphism of abstract schemes, but not of schemes over $\bar{k}$ or of varieties over $\bar{k}$.)

For example, assume, in addition, that $X, Y$, and the morphism $X \rightarrow Y$ are all defined over a number field $k$ in Lemma 3.1.1. Let $t$ be a point of $Y^{\circ}$ which has exact degree $d \geq 1$ over $k$ (i.e., $t \in Y^{\circ}(k,=d)$ ) and let $t^{(1)}=t, t^{(2)}, \ldots, t^{(d)}$ be the $d$ distinct Gal $(\bar{k} / k)$-conjugates of $t$, which are supposed to belong to $Y^{\circ}$ by the above fact. So every positive-dimensional closed subvariety of the fiber of the induced morphism $X^{(d)} \rightarrow Y^{(d)}$ over the point $\sum_{i=1}^{d}\left(t^{(i)}\right) \in Y^{(d)}$ has an effective canonical divisor.

In what follows, by $Y^{\circ}$ we always mean the set of $t$ 's $\in Y(\bar{k})$ such that the fiber $X_{t}$ over $t$ under the morphism $X \rightarrow Y$ is nonsingular and has the same dimension as that of the generic fiber of $X \rightarrow Y$, unless otherwise stated. In other words, it will be the case, even when we may have other various kinds of fibrations over $Y$, unless otherwise stated.

3.2. Proof of Theorem 1.0.1 Case $d=1$. We separate the two cases Case 1: $d=1$ and Case 2: $d \geq 2$. We will use induction on $d$, and we will often enlarge the number field $k$ according to our need. In particular, in the proof for Case $d \geq 2$, this simply means 'lowering' the degree of points under consideration, which makes sense by induction. Furthermore, the proof for Case 1 is easier to follow, and it may help us understand that for Case 2 more clearly.

By applying DHA to $h$, we may assume, whenever necessary in steps of our proof, that the height $h$ on $X$ is associated to an ample divisor of $X$ with $h \geq 0$ or 1. (Of course, we may start by assuming that $h \geq 1$ is associated to an ample divisor of $X$. However, to see the exact requirement to apply and to understand previous results better, we will indicate the places where we really need $h$ (or other heights) $\geq 1$.)

Proof of Theorem 1.0.1: Case $d=1$. We may assume, by enlarging $k$ if necessary, that the divisor $D$ and the canonical divisor of $X$ are also defined over $k$. Apply 
Proposition 2.5.1 to get

$$
h \leq O\left(h_{Y}(t)\right)
$$

on $X_{t} \cap \mathcal{R}-Z$, where $Z$ is a proper Zariski closed subset of $X$.

Hence we now need to deal with the points of $Z \cap \mathcal{R}$. Suppose that $Z$ may have several irreducible components and pick any one of them, say $B$. Let $Y_{0} \subset Y$ be the image of $B$ under $\pi$. If $Y_{0} \subset Y-Y^{\circ}$, then we are certainly done. And, if $B \subset \operatorname{Supp} D$, then we are also done, since $\mathcal{R}(\subset X(k, d)-\operatorname{Supp} D) \subset X-\operatorname{Supp} D$ by definition.

Thus, from now on, suppose that it is not either of the two cases. By Proposition 2.3 .1 we can find desingularizations $\widetilde{B}$ and $\widetilde{Y_{0}}$, respectively, of $B$ and $Y_{0}$ such that the following diagram

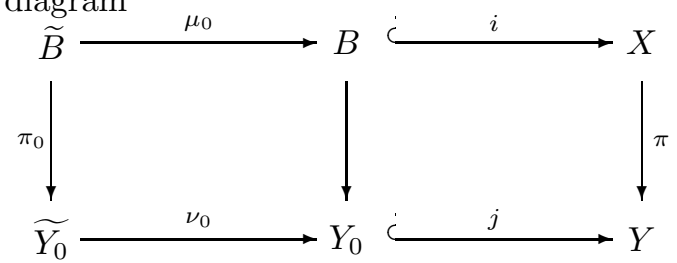

is commutative. We may assume, by enlarging $k$ if necessary, that all the varieties and the morphisms shown in this diagram are defined over $k$. If $\pi_{0}$ has relative dimension 0, i.e., $\operatorname{dim} \widetilde{B}=\operatorname{dim} \widetilde{Y_{0}}$, then we can rely on Theorem 2.3 .3 together with Example 2.3.2. So we exclude this possibility, too.

Notice that the birational morphism $\mu_{0}: \widetilde{B} \rightarrow B$ gives rise to an isomorphism

$$
\widetilde{B}-\mu_{0}^{-1}\left(B_{0}\right) \cong B-B_{0},
$$

where $B_{0}$ is a proper Zariski closed subset of $B$. (Notice that $B_{0}$ may be larger than the singular locus of $B$.) As usual, we may assume, by enlarging $k$ if necessary, that the isomorphism and its inverse are also defined over $k$.

Furthermore, we may assume, in addition, that (by abuse of notation)

$$
\mathcal{D}:=\operatorname{Supp} \mu^{*} D\left(=\left(\mu^{*} D\right)_{\text {reduced }}\right)
$$

$\left(\mu:=i \circ \mu_{0}\right)$ is a divisor with normal crossings. (Choose an appropriate blow-up.) Since $\mu(\widetilde{B})=B \not \subset$ Supp $D$ by hypothesis, $\mu^{*} D$ is an effective divisor of $\widetilde{B}$. (Also observe that $B \cap \operatorname{Supp} D \neq \emptyset$ since Supp $D$ maps onto $Y$ under $\pi$ by hypothesis.) We may assume, by enlarging $k$ if necessary, that $\mathcal{D}$ and the canonical divisor of $\widetilde{B}$ are also defined over $k$.

Let

$$
\mathfrak{p} \in \widetilde{B}(\bar{k}) \text { such that } \mu(\mathfrak{p}) \in \mathcal{R} .
$$

Since $\lambda_{\mu^{*} D, v}(\mathfrak{p})=\lambda_{D, v}(\mu(\mathfrak{p}))\left(v\right.$ a prime, finite or infinite, of $k$ - extend $|\cdot|_{v}$ to $(\bar{k} \hookrightarrow) \bar{k}_{v}$ as usual), it then follows, in particular, that $\mathfrak{p}$ is an $\left(S, \mu^{*} D\right)$-integral point of $\widetilde{B}$, whence an $(S, \mathcal{D})$-integral point, too. (Notice the multiplicities of $\mu^{*} D$ do not affect integrality. See [29, 1.4.4 (c) and 1.4.5, as well.) By the assumption on the field of definition for the isomorphism and its inverse in (2), we see that $\mathfrak{p} \in \widetilde{B}(k)$ if, in addition, $\mu(\mathfrak{p}) \in B-B_{0}$. (Notice that already $\mu(\mathfrak{p}) \in B \cap \mathcal{R} \subset B(k)$ since we are working in the case of $d=1$.)

Now apply Proposition 2.5.1 to $\pi_{0}$ and have: With any choice of a height $h_{\widetilde{B}}$ on $\widetilde{B}$ and a height $h_{\widetilde{Y_{0}}} \geq 1$ on $\widetilde{Y_{0}}$ associated to an ample divisor,

$$
h_{\widetilde{B}}(\mathfrak{p}) \leq O\left(h_{\widetilde{Y_{0}}} \circ \pi_{0}(\mathfrak{p})\right)
$$


whenever $\mu(\mathfrak{p}) \in \mathcal{R}-B_{1}$ where $B_{1}$ is a proper Zariski closed subset of $B$ containing $B_{0}$.

Let $\nu=j \circ \nu_{0}$ and recall Example 2.3.2 and Theorem 2.3.3. Furthermore, assume that $h_{\widetilde{B}}$ is associated to an ample divisor of $\widetilde{B}$ with $h_{\widetilde{B}} \geq 1$. For every such integral point $\mathfrak{p}$ of $\widetilde{B}(k)$ with $\mu(\mathfrak{p}) \in \mathcal{R}-B_{2}\left(B_{2}\right.$ a proper Zariski closed subset of $B$ containing $B_{1}$ ), we then have the following two inequalities together with (3) above:

$$
\begin{aligned}
h \circ \mu & \leq O\left(h_{\widetilde{B}}\right) ; \\
h_{\widetilde{Y_{0}}} \circ \pi_{0} & \leq O\left(h_{Y} \circ \nu \circ \pi_{0}\right) .
\end{aligned}
$$

Then combine (3), (4), and (5) to get the desired inequality

$$
h \leq O\left(h_{Y}(t)\right)
$$

on $X_{t} \cap B \cap \mathcal{R}-B_{2}$.

Now, in order to finish the proof for Case 1 , it only remains to repeat this process as often as needed.

Proof of Theorem 1.0.1: Case $d \geq 2$. Inevitably, many arguments will be very similar to those in the proof for Case 1. And, for convenience, we will use many of the same letters for their corresponding meanings (though not always). So let us take care not to be disturbed by the old notation too much.

In this section we recall, in particular, Example 2.1.1 and its notation. (In particular, apply it to the divisor $D$ for $H$.)

Apply Proposition 2.3.1 and have desingularizations $\mu: \widetilde{X^{(d)}} \rightarrow X^{(d)}$ and $\widetilde{Y^{(d)}} \rightarrow$ $Y^{(d)}$ such that $\pi^{(d)} \circ \mu: \widetilde{X^{(d)}} \rightarrow X^{(d)} \rightarrow Y^{(d)}$ is equal to $\widetilde{X^{(d)}} \stackrel{\pi_{0}}{\rightarrow} \widetilde{Y^{(d)}} \rightarrow Y^{(d)}$. We may assume, by enlarging $k$ if necessary, that all the varieties and the morphisms here are defined over $k$.

Notice that the birational morphism $\mu: \widetilde{X^{(d)}} \rightarrow X^{(d)}$ gives rise to an isomorphism

$$
\widetilde{X^{(d)}}-\mu^{-1}\left(Z_{0}\right) \cong X^{(d)}-Z_{0}
$$

where $Z_{0}$ is a proper Zariski closed subset of $X^{(d)}$. We may assume, by enlarging $k$ if necessary, that the isomorphism and its inverse are also defined over $k$.

In addition, we may assume, as before, that

$$
\mathcal{D}:=\operatorname{Supp} \mu^{*} \sigma_{*}\left(\sum_{i=1}^{d} \sigma_{i}^{*} D\right)\left(=\left(\mu^{*} \sigma_{*}\left(\sum_{i=1}^{d} \sigma_{i}^{*} D\right)\right)_{\text {reduced }}\right)
$$

(again by abuse of notation) is a divisor with normal crossings. We then also see that $\mu^{*} \sigma_{*}\left(\sum_{i=1}^{d} \sigma_{i}^{*} D\right)$ is an effective divisor (which is not the zero divisor). Furthermore, $\sigma_{*}\left(\sum_{i=1}^{d} \sigma_{i}^{*} D\right)$ being an ample divisor (cf. Example 2.1.1), $\mu^{*} \sigma_{*}\left(\sum_{i=1}^{d} \sigma_{i}^{*} D\right)$ is a big divisor and so is $\mathcal{D}$. We may assume, by enlarging $k$ if necessary, that both $\mathcal{D}$ and the canonical divisor $K_{\widetilde{X^{(d)}}}$ of $\widetilde{X^{(d)}}$ are also defined over $k$.

Note that

$$
Y^{\circ}(k, d)=\bigcup_{i=1}^{d} Y^{\circ}(k,=i) .
$$


Also we assume, by induction hypothesis, that the desired result is true up to $d-1$ (over an arbitrary number field $k$ ). Thus, from now on, we have only to deal with the points on $Y^{\circ}(k,=d)$.

Now let

$$
P \in \mathcal{R} \text { with } \pi(P)=: t \in Y^{\circ}(k,=d),
$$

hence $P \in X_{t}(k(t))$ is an $\left(S_{k(t)}, D_{t}\right)$-integral point (which, of course, belongs to $\left.X_{t}-\operatorname{Supp} D_{t}\right)$; cf. Section 2.4. Also, for $1 \leq i \leq d$, let

$$
t^{(i)} \in Y^{\circ}(k,=d)
$$

be the $d$ distinct Gal $(\bar{k} / k)$-conjugates of $t=t^{(1)}$ and let

$$
P^{(i)} \in X_{t^{(i)}}
$$

be the $d$ distinct Gal $(\bar{k} / k)$-conjugates of $P=P^{(1)}$. Clearly $P^{(i)} \in X_{t^{(i)}}\left(k\left(t^{(i)}\right)\right)$.

Let

$$
\mathfrak{p} \in \widetilde{X^{(d)}}(\bar{k})
$$

such that

$$
\mu(\mathfrak{p})=\mathcal{P}:=\sum_{i=1}^{d} P^{(i)}=\sigma\left(\left(P^{(1)}, \ldots, P^{(d)}\right)\right) \in X^{(d)}(k) .
$$

Note that $\mathcal{R}(\subset X(k, d)-\operatorname{Supp} D) \subset X-\operatorname{Supp} D$ by definition implies that

$$
\mathcal{P} \in X^{(d)}-\operatorname{Supp} \sigma_{*}\left(\sum_{i=1}^{d} \sigma_{i}^{*} D\right)
$$

i.e., that no $P^{(i)}$ belongs to $\operatorname{Supp} D$, since $D$ may be assumed to be defined over $k$ by enlarging $k$ if necessary. Thus it immediately follows that $\mu(\mathfrak{p}) \in X^{(d)}-$ Supp $\sigma_{*}\left(\sum_{i=1}^{d} \sigma_{i}^{*} D\right)$ and hence that

$$
\mathfrak{p} \in \widetilde{X^{(d)}}-\operatorname{Supp} \mu^{*} \sigma_{*}\left(\sum_{i=1}^{d} \sigma_{i}^{*} D\right)
$$

Let $v$ be a prime, finite or infinite, of $k_{1}$ where $k_{1}$ is a finite extension of the compositum of $k\left(t^{(i)}\right)$ 's over which there is a local equation $f$ of $D$. (As usual, we extend $|\cdot|_{v}$ to $\left(\overline{k_{1}} \hookrightarrow\right)\left(\overline{k_{1}}\right)_{v}$.) Then, noticing that $f\left(P^{(i)}\right)$ 's are conjugates and 
recalling Example 2.1.1, we observe that

$$
\begin{aligned}
& \lambda_{\mu^{*} \sigma_{*}\left(\sum_{i=1}^{d} \sigma_{i}^{*} D\right), v}(\mathfrak{p})=\lambda_{\sigma_{*}\left(\sum_{i=1}^{d} \sigma_{i}^{*} D\right), v}(\mu(\mathfrak{p})) \\
& =\lambda_{\sigma_{*}\left(\sum_{i=1}^{d} \sigma_{i}^{*} D\right), v}\left(\sigma\left(P^{(1)}, \ldots, P^{(d)}\right)\right) \\
& =\lambda_{\sigma^{*} \sigma_{*}\left(\sum_{i=1}^{d} \sigma_{i}^{*} D\right), v}\left(P^{(1)}, \ldots, P^{(d)}\right) \\
& =c \cdot \lambda_{\sum_{i=1}^{d} \sigma_{i}^{*} D, v}\left(P^{(1)}, \ldots, P^{(d)}\right) \\
& =c \cdot \sum_{i=1}^{d} \lambda_{\sigma_{i}^{*} D, v}\left(P^{(1)}, \ldots, P^{(d)}\right) \\
& =c \cdot \sum_{i=1}^{d} \lambda_{D, v}\left(\sigma_{i}\left(P^{(1)}, \ldots, P^{(d)}\right)\right) \\
& =c \cdot \sum_{i=1}^{d} \lambda_{D, v}\left(P^{(i)}\right) \\
& =c \cdot \sum_{i=1}^{d}\left(-\frac{1}{\left[k_{1}: \mathbb{Q}\right]} \log \left|f\left(P^{(i)}\right)\right|_{v}+\alpha_{D, v}\left(P^{(i)}\right)\right) \\
& =c \cdot \sum_{i=1}^{d}\left(-\frac{1}{\left[k_{1}: \mathbb{Q}\right]} \log |f(P)|_{v}+\alpha_{D, v}\left(P^{(i)}\right)\right) \\
& =c \cdot \sum_{i=1}^{d}\left(\left\{\left\{-\frac{1}{\left[k_{1}: \mathbb{Q}\right]} \log |f(P)|_{v}+\alpha_{D, v}(P)\right\}\right.\right. \\
& \left.\left.-\alpha_{D, v}(P)\right\}+\alpha_{D, v}\left(P^{(i)}\right)\right) \\
& =c \cdot \sum_{i=1}^{d}\left(\left\{\lambda_{D, v}(P)-\alpha_{D, v}(P)\right\}+\alpha_{D, v}\left(P^{(i)}\right)\right) \\
& =c \cdot \sum_{i=1}^{d}\left(\lambda_{D, v}^{\prime}(P)+\alpha_{D, v}\left(P^{(i)}\right)\right) \\
& =c \cdot d \cdot \lambda_{D, v}^{\prime}(P)+c \cdot \sum_{i=1}^{d} \alpha_{D, v}\left(P^{(i)}\right),
\end{aligned}
$$

where $\lambda_{D, v}^{\prime}:=\lambda_{D, v}-\alpha_{D, v}$, which we should notice is also a Weil function. Note that $\alpha_{D, v}$ is the " $v$-component" of a locally bounded continuous function associated to $D$. For its detail, see [16] or 29]. Although we always carried $\alpha_{D, v}$ in the meantime to see a general phenomenon, we may put $\alpha_{D, v}=0$ (in (18) and) (99)) in the light of [29], 1.4.4 (c) and 1.4.5. Consequently, this amounts to enabling us to put $\alpha_{\mu^{*} \sigma_{*}\left(\sum_{i=1}^{d} \sigma_{i}^{*} D\right), v}=0$ (in the same spirit).

We see (from (10) in the same spirit as above) that $\mathfrak{p}$ is an $\left(S, \mu^{*} \sigma_{*}\left(\sum_{i=1}^{d} \sigma_{i}^{*} D\right)\right)$ integral point of $\widetilde{X^{(d)}}$ (which belongs to $\widetilde{X^{(d)}}-\mathcal{D}$ ), hence an $(S, \mathcal{D})$-integral point, too. Also, by the assumption on the field of definition for the isomorphism and its inverse in (7), we see that $\mathfrak{p} \in \widetilde{X^{(d)}}(k)$ if, in addition, $\mu(\mathfrak{p}) \in X^{(d)}-Z_{0}$. (Notice that we already have $\mu(\mathfrak{p})=\mathcal{P} \in X^{(d)}(k)$.) 
Now apply Proposition 2.5.1 to $\pi_{0}: \widetilde{X^{(d)}} \rightarrow \widetilde{Y^{(d)}}$ and get: With any choice of a height $h_{\widetilde{X^{(d)}}}$ on $\widetilde{X^{(d)}}$ and a height $h_{\widetilde{Y^{(d)}}} \geq 1$ on $\widetilde{Y^{(d)}}$ associated to an ample divisor,

$$
h_{\widetilde{X^{(d)}}}(\mathfrak{p}) \leq O\left(h_{\widetilde{Y^{(d)}}} \circ \pi_{0}(\mathfrak{p})\right)
$$

whenever $\mu(\mathfrak{p}) \in X^{(d)}-Z_{1}$, where $Z_{1}$ is a proper Zariski closed subset of $X^{(d)}$ containing $Z_{0}$.

Now, furthermore, assume that $h_{\widetilde{X^{(d)}}}$ is associated to an ample divisor of $\widetilde{X^{(d)}}$ with $h_{\widetilde{X^{(d)}}} \geq 1$. Recall how to get (6) from the combination of (3), (4) , and (5). Then an exact analogous argument with the obvious extra help of Example 2.1.1, (3) will yield

$$
h(P) \leq O\left(h_{Y}(t)\right)
$$

for all $P \in X_{t} \cap \mathcal{R}$ such that $\mathcal{P} \in X^{(d)}-Z$, where $Z$ is a proper Zariski closed subset of $X^{(d)}$ containing $Z_{1}$. (We omit the repetition of its details.)

Hence we now need to deal with the points $P \in \mathcal{R}$ such that $\mathcal{P} \in Z$. Suppose that $Z$ may have several irreducible components and pick any one of them, say $B$. Let

$$
Y_{0} \subset Y^{(d)}
$$

be the image of $B$ under $\pi^{(d)}: X^{(d)} \rightarrow Y^{(d)}$. If $B \subset \operatorname{Supp} \sigma_{*}\left(\sum_{i=1}^{d} \sigma_{i}^{*} D\right)$, then we are done. For $\mathcal{R}(\subset X(k, d)-\operatorname{Supp} D) \subset X-\operatorname{Supp} D$ by definition, which implies that $\mathcal{P} \in X^{(d)}-\operatorname{Supp} \sigma_{*}\left(\sum_{i=1}^{d} \sigma_{i}^{*} D\right)$, i.e., that no $P^{(i)}$ belongs to Supp $D$, since $D$ may be assumed to be defined over $k$ by enlarging $k$, if necessary, as before. (See the paragraph preceding (8) above, too.)

From now on, suppose that it is not the case. Apply Proposition 2.3.1 to have desingularizations $\widetilde{B}$ and $\widetilde{Y_{0}}$ of $B$ and $Y_{0}$, respectively, such that the diagram

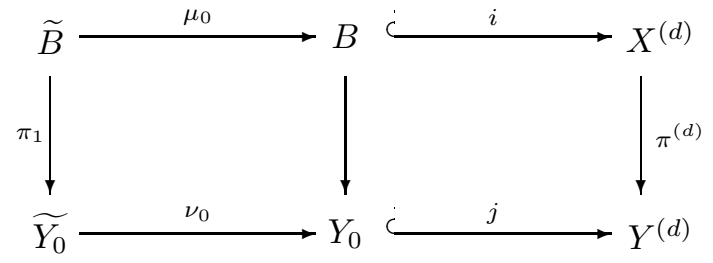

is commutative. We may assume, by enlarging $k$ if necessary, that all the varieties and the morphisms shown in this diagram are defined over $k$. If $\pi_{1}$ has relative dimension 0, then we can rely on Example 2.3.2 and Theorem 2.3.3 together with the obvious extra help of Example 2.1.1. (3). So we exclude this possibility, too.

Notice that the birational morphism $\mu_{0}: \widetilde{B} \rightarrow B$ gives rise to an isomorphism

$$
\widetilde{B}-\mu_{0}^{-1}\left(B_{0}\right) \cong B-B_{0},
$$

where $B_{0}$ is a proper Zariski closed subset of $B$. As usual, we may assume, by enlarging $k$ if necessary, that the isomorphism and its inverse are also defined over $k$.

Furthermore, as before, we may assume, in addition, that

$$
\mathcal{D}_{1}:=\operatorname{Supp} \mu_{1}^{*} \sigma_{*}\left(\sum_{i=1}^{d} \sigma_{i}^{*} D\right)\left(=\left(\mu_{1}^{*} \sigma_{*}\left(\sum_{i=1}^{d} \sigma_{i}^{*} D\right)\right)_{\text {reduced }}\right)
$$


$\left(\mu_{1}:=i \circ \mu_{0}\right)$ is a divisor with normal crossings. Since

$$
\mu_{1}(\widetilde{B})=B \not \subset \operatorname{Supp} \sigma_{*}\left(\sum_{i=1}^{d} \sigma_{i}^{*} D\right)
$$

by hypothesis, $\mu_{1}^{*} \sigma_{*}\left(\sum_{i=1}^{d} \sigma_{i}^{*} D\right)$ is an effective divisor of $\widetilde{B}$ (which is not the zero divisor). Also, we know that $\mu_{1}^{*} \sigma_{*}\left(\sum_{i=1}^{d} \sigma_{i}^{*} D\right)$ is a big divisor and hence that so is $\mathcal{D}_{1}$. We may assume, by enlarging $k$ if necessary, that $\mathcal{D}_{1}$ and the canonical divisor $K_{\widetilde{B}}$ of $\widetilde{B}$ are also defined over $k$.

Then, as before (cf. (8) and (10)), we see that $\mathfrak{p} \in \widetilde{B}$ with $\mu_{1}(\mathfrak{p})=\mathcal{P}$ must be an $\left(S, \mu_{1}^{*} \sigma_{*}\left(\sum_{i=1}^{d} \sigma_{i}^{*} D\right)\right.$ )-integral point of $\widetilde{B}$ (which belongs to $\widetilde{B}-\mathcal{D}_{1}$ ), hence an $\left(S, \mathcal{D}_{1}\right)$-integral point, too. Also, as before, with the aid of the assumption on the field of definition for the isomorphism and its inverse in (13), we see that $\mathfrak{p} \in \widetilde{B}(k)$ if, in addition, $\mu_{1}(\mathfrak{p}) \in B-B_{0}$. (Note that already $\mu_{1}(\mathfrak{p})=\mathcal{P} \in B(k)$.)

Now apply Proposition 2.5.1 to $\pi_{1}: \widetilde{B} \rightarrow \widetilde{Y_{0}}$ and get: With any choice of a height $h_{\widetilde{B}}$ on $\widetilde{B}$ and a height $h_{\widetilde{Y_{0}}} \geq 1$ on $\widetilde{Y_{0}}$ associated to an ample divisor,

$$
h_{\widetilde{B}}(\mathfrak{p}) \leq O\left(h_{\widetilde{Y_{0}}} \circ \pi_{1}(\mathfrak{p})\right)
$$

whenever $\mu(\mathfrak{p}) \in B-B_{1}$, where $B_{1}$ is a proper Zariski closed subset of $B$ containing $B_{0}$.

Then, exactly similar to the way to have (12) together with the extra assumption that $h_{\widetilde{B}}$ is associated to an ample divisor of $\widetilde{B}$ with $h_{\widetilde{B}} \geq 1$, it follows that

$$
h(P) \leq O\left(h_{Y}(t)\right)
$$

for all $P \in X_{t} \cap \mathcal{R}$ such that $\mathcal{P} \in X^{(d)} \cap B-B_{2}$, where $B_{2}$ is a proper Zariski closed subset of $B\left(\subset Z \subset X^{(d)}\right)$ containing $B_{1}$. (We also omit its details.)

Now it remains only to repeat this process as often as needed. Therefore we conclude the proof for Case 2 and the whole proof of Theorem 1.0.1

\section{Miscellanea}

4.1. Slightly strengthened version of Theorem 1.0.1, We combine Example 2.3.2 and Theorem 1.0.1 to get:

Theorem 4.1.1. Keep the hypotheses of Theorem 1.0.1 and let $h \geq 1$ (resp. $h_{Y} \geq$ 1 ) be a height on $X$ (resp. $Y$ ) associated to an ample divisor of $X$ (resp. $Y)$. Then, assuming the Vojta conjecture for varieties of dimension $\leq d \cdot \operatorname{dim} X$, we have

$$
h(P) \gg \ll h_{Y}(\pi(P)),
$$

for all $P \in \mathcal{R}$, where the implied constants are independent of $P$.

4.2. What more can be said? As should be already noted, essentially the same corresponding proofs will give the following results. (We omit the easy necessary change of arguments.)

In this section we agree: Whatever $X$ and $Y$ are, $h$ is an arbitrary height on $X$ and $h_{Y}$ is a height on $Y$ associated to an ample divisor satisfying $h_{Y} \geq 1$.

First, regarding integral points on abelian varieties: Let $\pi: X \rightarrow Y$ be a family of abelian varieties, i.e., both $X$ and $Y$ are nonsingular projective varieties (of arbitrary dimension) and all the nonsingular fibers are abelian varieties - the 
generic fiber is an abelian variety. Assume, in addition, that $X, Y$, and $\pi$ are all defined over a number field $k$ and let $S$ be a finite set of primes of $k$ containing all the infinite ones.

Let $d \geq 1$ be an integer. Let $D$ be an ample divisor (assumed to be effective) of $X$ with normal crossings. (So it is not fibral, i.e., $\pi(\operatorname{Supp} D)=Y$.) Denote by

$$
\mathcal{R} \subset X(k, d)-\operatorname{Supp} D
$$

a subset of $(S, D)$-integral points of $X(\bar{k})$ which belong to $X(k, d)-\operatorname{Supp} D$.

Theorem 4.2.1. In addition, assume the Vojta conjecture for varieties of dimension $\leq d \cdot \operatorname{dim} X$. Then we have

$$
h(P) \leq O\left(h_{Y}(\pi(P))\right)
$$

for all $P \in \mathcal{R}$, i.e., $P$ an $\left(S_{\pi(P)}, D_{\pi(P)}\right)$-integral point of $X_{\pi(P)}(k(\pi(P)))$, with $\pi(P) \in Y^{\circ}(k, d)$, where the implied constant is independent of $P$.

Here we invoke Faltings' result ([7]) that asserts the finiteness of integral points on an abelian variety outside (the support of) an ample divisor and that also answers a conjecture of Lang's.

Second, regarding integral points on rational curves: Let $\pi: X \rightarrow$ $Y$ be a family of rational curves, i.e., both $X$ and $Y$ are nonsingular projective varieties (of arbitrary dimension), and all the 1-dimensional nonsingular fibers of the surjective morphism $\pi$ of relative dimension 1 are rational curves - the generic fiber is isomorphic to the projective line $\mathbb{P}^{1}$. Assume, in addition, that $X, Y$, and $\pi$ are all defined over a number field $k$ and let $S$ be as above.

Let $D$ be an ample divisor (assumed to be effective) of $X$ with normal crossings such that Supp $D$ meets each $X_{t}\left(t \in Y^{\circ}\right)$ in at least three distinct points. Let

$$
\mathcal{R} \subset X(k)-\operatorname{Supp} D
$$

be a subset of $(S, D)$-integral points of $X(\bar{k})$ which belong to $X(k)$ (thus, to $X(k)-$ Supp D).

Theorem 4.2.2. In addition, assume the Vojta conjecture for varieties of dimension $\leq \operatorname{dim} X$. Then we have

$$
h(P) \leq O\left(h_{Y}(\pi(P))\right)
$$

for all $P \in \mathcal{R}$, i.e., $P$ an $\left(S, D_{\pi(P)}\right)$-integral point of $X_{\pi(P)}(\bar{k})$ belonging to $X_{\pi(P)}(k)$, with $\pi(P) \in Y^{\circ}(k)$, where the implied constant is independent of $P$.

4.3. Height zeta function. Finally, we define height zeta function associated to families which we have considered, as follows:

Definition. Under the situation of Theorem 1.0.1 with the extra hypothesis that $S$ contains 3 (for convenience, with the assumption of $d=1$ ), we define a height zeta function

$$
\zeta_{X}(s)=\sum_{P \in X^{*}(k)} \frac{H(P)}{H_{Y}(\pi(P))^{s}}
$$

for $s \in \mathbb{C}$ with $\operatorname{Re} s \gg 0$, where $X^{*}$ means the union of stably $(S, D)$-integral points on nonsingular fibers and $H$ (resp. $H_{Y}$ ) is the exponential height corresponding to $h$ (resp. $h_{Y}$ ). Note, despite its abbreviated notation, that $\zeta_{X}$ actually depends on the choice of appearing heights as well as on $X, Y, D, S, \pi$, and $k$. 
Claim 4.3.1. $\zeta_{X}$ defines an analytic function for $s \in \mathbb{C}$ with $\operatorname{Re} s \gg 0$.

Proof. We may assume that $h_{Y} \geq 1$ is associated to a very ample divisor, by means of which we embed $Y \hookrightarrow \mathbb{P}^{N}$. We enlarge $k$ (if necessary) so that the embedding is also defined over $k$. Then we see that the height $h_{Y}$ is just the pull-back of the standard (logarithmic) height of $\mathbb{P}^{N}$ up to constant multiplication.

Regard $t:=\pi(P) \in Y^{\circ}(k)$ as a point of $\mathbb{P}^{N}(k)$ and apply Schanuel's formula for $\mathbb{P}^{N}(k)$. Then we have: For all integers $n \geq 1$,

$$
\left|\left\{t \in Y(k): n \leq H_{Y}(t) \leq n+1\right\}\right| \ll(n+1)^{(N+1)[k: \mathbb{Q}]} \ll n^{(N+1)[k: \mathbb{Q}]} .
$$

Let $\sigma=\operatorname{Re} s$ and let $c$ be the positive constant appearing in Theorem 1.0.1. Then

$$
\begin{aligned}
\sum_{P \in X^{*}(k)}\left|\frac{H(P)}{H_{Y}(\pi(P))^{s}}\right| & =\sum_{P \in X^{*}(k)} \frac{H(P)}{H_{Y}(\pi(P))^{\sigma}} \\
& =\sum_{P \in X^{*}(k)} \frac{1}{H_{Y}(\pi(P))^{\sigma-c}} \cdot \frac{H(P)}{H_{Y}(\pi(P))^{c}} \\
& \leq \sum_{P \in X^{*}(k)} \frac{1}{H_{Y}(\pi(P))^{\sigma-c}} \\
& \ll \sum_{t \in Y^{\circ}(k)} \frac{1}{H_{Y}(t)^{\sigma-c}} \text { by [22] } \\
& \ll \sum_{n=1}^{\infty} \frac{n^{(N+1)[k: \mathbb{Q}]}}{n^{\sigma-c}} \text { by (1) } \\
& =\sum_{n=1}^{\infty} \frac{1}{n^{\sigma-c-(N+1)[k: \mathbb{Q}]} .}
\end{aligned}
$$

Therefore $\zeta_{X}(s)$ is an analytic function for $\sigma>c+(N+1)[k: \mathbb{Q}]+1$.

Notice that we need to take only stably integral points on nonsingular fibers to define the above height zeta function, since we use [22] in the proof of Claim 4.3.1. A good question at this stage may be how we can derive some information about the constant $c>0$ appearing in Theorem 1.0.1 from any analytic study, e.g., using the Tauberian theorem, of the height zeta function above. It is yet to do.

\section{ACKNOWLEDGMENTS}

The author thanks the referee for his or her many helpful comments and suggestions.

\section{REFERENCES}

1. D. Abramovich, Uniformité des points rationnels des courbes algébriques sur les extensions quadratiques et cubiques, C. R. Acad. Sci. Paris Sér. I Math. 321 (1995), 755-758. MR.1354720 (96g:14017)

2. Uniformity of stably integral points on elliptic curves, Invent. Math. 127 (1997), no. 2, 307-317. MR1427620 (98d:14033)

3. D. Abramovich, and J. Harris Abelian varieties and curves in $W_{d}(C)$, Compositio Math. 78 (1991), 227-238. MR.1104789 (92c:14022) 
4. V. V. Batyrev, and Yu. I. Manin, Sur le nombre des points rationnels de hauteur borné des variétiés algébriques, Math. Ann. 286 (1990), 27-43. MR1032922 (91g:11069)

5. G. Call, and J. H. Silverman, Canonical heights on varieties with morphisms, Compos. Math. 89 (1993), 163-205. MR1255693 (95d:11077)

6. L. Caporaso, J. Harris, and B. Mazur, Uniformity of rational points, J. Amer. Math. Soc., vol. 10, no. 1 (Jan., 1997), 1-35. MR1325796 (97d:14033)

7. G. Faltings, Diophantine approximation on abelian varieties, Ann. of Math. (2) 133 (1991), 549-576. MR1109353 (93d:11066)

8. J. Franke, Yu. I. Manin, and Yu. Tschinkel, Rational points of bounded height on Fano varieties, Invent. Math. 95 (1989), no. 2, 421-435. (Erratum: Invent. Math. 102 (1990), no. 2, 463.) MR0974910 (89m:11060) MR1074483 (91i:11068)

9. W. Fulton, Intersection theory, Ergebnisse der Mathematik und ihrer Grenzgebiete (3) [Results in Mathematics and Related Area (3)], 2. Springer-Verlag, Berlin, 1984, xi+470 pp. MR:0732620 (85k:14004)

10. J. Harris, and J. Silverman, Bielliptic curves and symmetric products, Proc. Amer. Math. Soc. 112 (1991), no. 2, 347-356. MR 1055774 (91i:11067)

11. R. Hartshorne, Algebraic geometry, Graduate Texts in Mathematics, vol. 52, Springer-Verlag, New York, 1977. MR0463157(57:3116)

12. M. Hindry, Autour d'une conjecture de Serge Lang, Invent. Math. 94 (1988), 575-603. MR0969244 (89k:11046)

13. M. Hindry, and J. Silverman, Diophantine geometry. An introduction. Graduate Texts in Mathematics, 201, Springer-Verlag, New York, 2000. xiv+558 pp. MR.1745599|(2001e:11058)

14. H. Hironaka, Resolution of singularities of an algebraic variety over a field of characteristic O, I, II, Ann. of Math. (2), 79 (1964), 109-203; ibid. (2), 79 (1964), 205-326. MR0199184 (33:7333)

15. S.-I. Ih, Height uniformity for algebraic points on curves, Compositio Math. 134 (2002), 35-57. MR.1931961 (2004g:11052)

16. S. Lang, Fundamentals of Diophantine Geometry, Springer-Verlag, New York, 1983. MR0715605 (85j:11005)

17. 60 Springer-Verlag, Berlin, 1991. xiv+296 pp. MR1112552 (93a:11048)

18. J. S. Milne, Jacobian varieties, in "Arithmetic Geometry" (G. Cornell and J. H. Silverman, eds.), 167-212, Springer-Verlag, New York/Berlin, 1986. MR0861976

19. C. F. Osgood, A number theoretic-differential equations approach to generalizing Nevanlinna theory, Indian J. Math. 23 (1981), no. 1-3, 1-15. MR0722894 (85b:30043)

20. - Sometimes effective Thue-Siegel-Roth-Schmidt-Nevanlinna bounds, or better, J. Number Theory 21 (1985), no. 3, 347-389. MR0814011 (87f:11046)

21. P. L. Pacelli, Uniform boundedness for rational points, Duke Math. J. vol. 88 (1997), no. 1, 77-102. MR:1448017 (98b:14020)

22. _ Uniform bounds for stably integral points on elliptic curves, Proc. Amer. Math. Soc. 127 (1999), no. 9, 2535-2546. MR1676288 (99m:11065)

23. J-P. Serre, Lectures on the Mordell-Weil theorem, Asp. Math. E 15, Vieweg, 1989. MR.1002324 (90e:11086)

24. Algebraic groups and class fields, Translated from the French, Graduate Texts in Mathematics, 117, Springer-Verlag, New York-Berlin, 1988. x+207 pp. MR0918564 (88i:14041)

25. J. H. Silverman, Heights and the specialization map for families of abelian varieties, J. Reine, Angew. Math. 342 (1983), 197-211. MR0703488 (84k:14033)

26. - The arithmetic of elliptic curves, Graduate Texts in Mathematics, vol. 106, SpringerVerlag, New York, 1986. MR0817210 (87g:11070)

27. _ Rational points on symmetric products of a curve, Amer. J. Math. 113 (1991) 471508. MR:1109348 (92m:11060)

28. - Advanced topics in the arithmetic of elliptic curves, Graduate Texts in Mathematics, vol. 151, Springer-Verlag, New York, 1994. MR1312368 (96b:11074)

29. P. Vojta, Diophantine approximations and value distribution theory, Lecture Notes in Mathematics, vol. 1239, Springer-Verlag, New York, 1987. MR0883451 (91k:11049) 
30. - Arithmetic discriminants and quadratic points on curves, Arithmetic Algebraic Geometry, Texel 1989 (G. van der Geer, F. Oort, and J. H. M. Steenbrink, eds.), Prog. in Math., vol. 89, Birkhäuser, Boston (1991), 359-376. MR.1085268 (92j:11059)

31. _ Siegel's theorem in the compact case, Ann. of Math. (2) 133 (1991) 509-548. MR.1109352 (93d:11065)

Department of Mathematics, University of Georgia, Athens, Georgia 30602-7403

E-mail address: ih@math.uga.edu

Current address: Department of Mathematics, University of Colorado, Campus Box 395, Boulder, Colorado 80309-0395 